\title{
Índices de vegetación y unidades de paisaje de la Reserva de Fauna Silvestre Ciénaga de La Palmita e Isla de Pájaros, estado Zulia, Venezuela
}

\author{
Vegetation indices and landscape units of the Wildlife Fauna Reservoir Ciénaga de La Palmita \\ and Isla de Pájaros, Zulia state, Venezuela \\ Índices de vegetação e unidades paisagísticas da Reserva Natural de Ciénaga da Palmita e Ilha de \\ Pájaros, estado de Zulia, Venezuela
}

\author{
Antonio Vera \\ ajvera68@gmail.com \\ Gustavo Morillo \\ morillogustavo@hotmail.com \\ Darisol Pacheco \\ dpacheco@luz.edu.ve
}

Universidad del Zulia, Venezuela

Artículo recibido enero 2020, arbitrado febrero 2020 y publicado en mayo 2020

\section{RESUMEN}

Se determinaron los índices de vegetación y las unidades de paisaje de la Reserva de Fauna Silvestre Ciénaga de La Palmita e Isla de Pájaros, empleando imágenes LANDSAT 8 (2013-2016) y el NDVI usando el programa ENVI. Se identificaron siete unidades de paisaje. El espejo de agua mostró un NDVI bajo $(0,00-0,03)$, asociado al muy escaso vigor vegetal y el borde ciénaga-porción terrestre presentó 0,04-0,08 vinculado a los primeros indicios de productividad. Los suelos fangosos-desnudos revelaron 0,09-0,16 relacionado a escasas capas de fitoplancton. Las comunidades xerófilas intervenidas presentaron 0,17-0,24 contrastando con 0,25-0,28 de las formaciones xerófilas, espinosas y semideciduas bajas. Las comunidades de Avicennia germinans, Conocarpus erectus y Laguncularia racemosa mostraron 0,29-0,39 debido al bajo porte e individuos muertos; y Rhizophora mangle reveló $0,4-0,55$, vinculado con la altura arborea, dosel semicerrado, pocos claros y verdor foliar intensobrillante. Las unidades variaron desde comunidades xerófilas intervenidas hasta comunidades de R. mangle relativamente bien conservadas.

Palabras clave: NDVI; humedal; manglar; vegetación xerófila; perturbaciones antropogénicas; Lago de Maracaibo; sensores remotos

\begin{abstract}
Vegetation indices and landscape units of the Wildlife Fauna Reservoir Ciénaga de La Palmita and Isla de Pájaros were determined, using LANDSAT 8 images (2013-2016) and the NDVI using the ENVI program. Seven landscape units were identified. The water surface of the swamp showed a low NDVI (0.00-0.03), associated with very little plant vigor and the swamp-land portion presented 0.04-0.08 linked to first signs of productivity. Muddy-bare soils revealed 0.09-0.16 related to scarce layers of phytoplankton. Disturbed xerophytic communities presented $0.17-0.24$, contrasting with $0.25-0.28$ of the xerophytic, spiny and semi-deciduous formations. The communities of Avicennia germinans, Conocarpus erectus and Laguncularia racemosa showed 0.29-0.39 due to low size and dead individuals; and Rhizophora mangle revealed 0.40.55 , linked to arborea height, semi-enclosed canopy, few gaps and intense-bright leaf greenery. The units ranged from disturbed xerophytic scrubs to relatively well preserved R. mangle communities
\end{abstract}

Key words: NDVI; wetland; xerophytic vegetation; mangrove; Lake of Maracaibo; anthropogenic disturbances; remote sensing 


\section{RESUMO}

Os índices de vegetação e as unidades de paisagem da Reserva de Vida Selvagem Ciénaga, da Fauna da Palmita e Ilha de Pájaros foram determinados usando imagens do LANDSAT 8 (2013-2016) e o NDVI usando o programa ENVI. Sete unidades de paisagem foram identificadas. 0 corpo de água apresentou baixo NDVI (0,00-0,03), associado a um vigor muito baixo das plantas, e a borda da porção de pântano-terra apresentou $0,04-0,08$, associada aos primeiros sinais de produtividade. Os solos lamacentos revelaram 0,09-0,16, relacionados às camadas esparsas de fitoplâncton. As comunidades xerófitas intervenientes apresentaram 0,17-0,24, contrastando com 0,25-0,28 para as formações xerófilas, espinhosas e baixas semidecíduas. As comunidades de Avicennia germinans, Conocarpus erectus e Laguncularia racemosa apresentaram 0,29-0,39 devido ao seu baixo tamanho e indivíduos mortos; e Rhizophora mangle revelaram 0,4-0,55, ligados a altas árvores, copa semi-fechada, poucas brechas e vegetação de folha intensa e brilhante. As unidades variaram de comunidades xerófitas intervenientes a comunidades de R. mangle relativamente bem preservadas

Palavras-chave: NDVI; pantanal; manguezal; vegetação xerofítica; distúrbios antropogênicos; Lago de Maracaibo; sensoriamento remoto

\section{INTRODUCCIÓN}

Las investigaciones sobre las comunidades vegetales en Venezuela son de gran importancia dado que aportan conocimiento novedoso sobre la fitogeografía nacional, permitiendo identificar los principales agentes tensores y perturbaciones que amenazan hoy en día la biodiversidad vegetal del país y la vida silvestre en general.

En el caso del estado Zulia, localizado al occidente de Venezuela, se ha presentado una tasa elevada de deforestación durante los últimos 30 años, a lo que se adiciona el desconocimiento que existe sobre la florística y estructura de las comunidades forestales primarias. Aunado a ello, el sector oriental de la cuenca del Lago de Maracaibo constituye una de las zonas más desconocidas en cuanto a su vegetación (Pietrangeli et al., 2011).

La Ciénaga de La Palmita se localiza en la costa oriental del Estrecho del Lago de Maracaibo, y la misma ha estado sometida a diferentes impactos como el déficit de la precipitación, la casi desaparición o reducción drástica del suministro de agua del río Aurare, la ocupación y expansión urbana, la reducción de la escorrentía, la deposición de desechos sólidos entre otros, que amenazan y comprometen su estabilidad $\mathrm{y}$ funcionamiento ecosistémico (Medina $\mathrm{y}$ Barboza, 2006).

Sin embargo, y a pesar de las condiciones actuales, la Ciénaga de La Palmita fue declarada por el estado venezolano Área Bajo Régimen de Administración Especial (ARAE) con fines protectores el 09 de Marzo del 2000, bajo la denominación de Reserva de Fauna Silvestre Ciénaga de La Palmita e Isla de Pájaros y el objetivo de tal declaratoria fue la conservación del hábitat de numerosas especies de aves, especies de interés cinegético y especies en peligro de extinción (Gaceta Oficial de la República Bolivariana de Venezuela, 2000).

Esta reserva de fauna posee un área de $2.525,85$ ha repartidas en cuatro espacios fisiográficos, tres de ellos continentales: la ciénaga propiamente dicha, el bosque xerófilo y el bosque de manglar, y uno insular: la isla de Pájaros (Gaceta Oficial de la República Bolivariana de Venezuela, 2000).

Aunque la Reserva de Fauna Silvestre (RFS) en estudio está integrada por estos importantes ambientes naturales, son muy escasas las investigaciones científicas realizadas en esta área natural protegida (De Olivares, 1988; Coty, 1995; González y Borjas, 2003; Vera et al., 2010; Vera et al., 2011a; Vera et al., 2011b; Vera et al., 2014). 
Las diversas perturbaciones a las que ha estado sometida esta reserva de fauna silvestre y los escasos trabajos científicos llevados a cabo en la misma han motivado el uso de las imágenes satelitales LANDSAT 8 (sensores remotos) y del Índice Normalizado de Diferencias en la Vegetación (NDVI por sus siglas en inglés).

En este sentido, Powel et al. (2014) han señalado que las imágenes satelitales LANDSAT 8 son ampliamente utilizadas para el cálculo de índices de vegetación ya que a partir de estas se obtienen buenos resultados. De igual manera, Meera Gandhi et al. (2015) han indicado que el NDVI es un índice muy importante y ampliamente utilizado en el monitoreo de la vegetación, destacando la clasificación de la cobertura vegetal, de los tipos de bosques y de las comunidades forestales.

Todos estos argumentos han conducido a combinar ambas herramientas metodológicas para obtener datos sobre los tipos de formaciones vegetales existentes así como el estado de conservación y/o intervención en que se encuentran éstas, dado que no se conoce dicha información para la Reserva de Fauna Silvestre investigada.

Además de ello, estos tipos de estudios son relativamente escasos en la región zuliana, destacando la investigación sobre la detección de 75 fragmentos de remanentes de bosques secos a partir de imágenes satelitales
LANDSAT TM 2003 para la Planicie de Maracaibo, de la que se obtuvieron valores de NDVI de 0,56 y 0,89 para las épocas de sequía y lluviosa respectivamente (Valbuena et al., 2011). Recientemente Morillo Díaz et al. (2018) determinaron productividad primaria en el Lago de Maracaibo a partir de imágenes LANDSAT 8 del periodo 2011-2016 y se calcularon los índices de vegetación NDVI y NRVI.

El objetivo de esta investigación es determinar los índices de vegetación y las unidades de paisaje de la Reserva de Fauna Silvestre Ciénaga de La Palmita e Isla de Pájaros, estado Zulia, Venezuela.

\section{MATERIALES Y MÉTODOS}

\section{Área de estudio}

La Reserva de Fauna Silvestre Ciénaga de La Palmita e Isla de Pájaros está situada en el estrecho del Lago Maracaibo, estado Zulia entre $10^{\circ}-35^{\prime} 12^{\prime \prime}-10^{\circ} 38^{\prime} 23^{\prime \prime} \mathrm{N}$ y $71^{\circ} 26^{\prime} 41^{\prime \prime}$ -

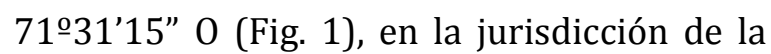
parroquia Santa Rita del municipio del mismo nombre y de las parroquias Ana María Campos y Altagracia del municipio Miranda, (Gaceta Oficial de la República Bolivariana de Venezuela, 2000), en el subsector semiárido de la región norte de la costa oriental del Lago, en zona de vida de bosque muy seco tropical según Ewel y Madriz (1968). 

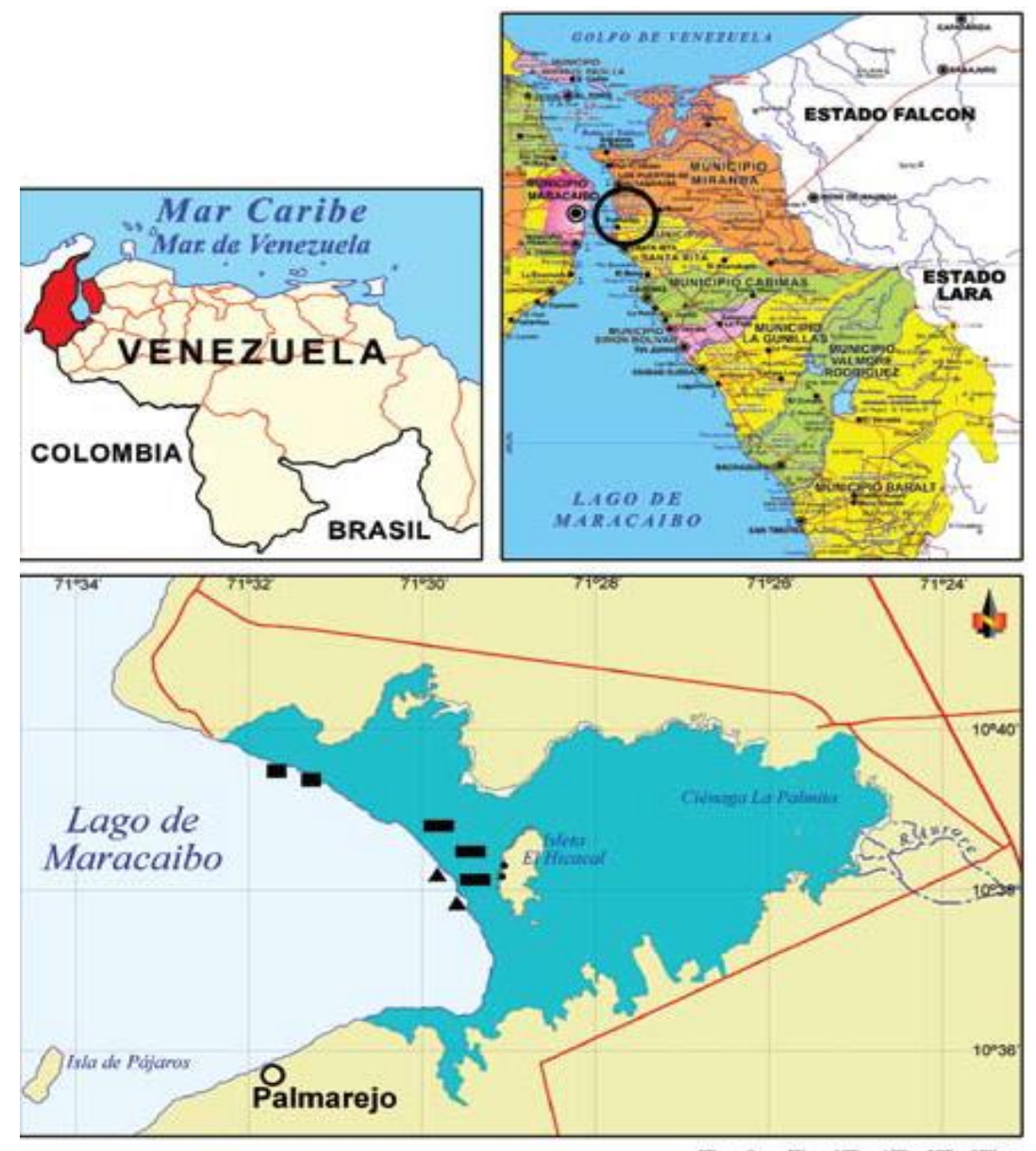

Figura 1. Área de estudio Reserva de Fauna Silvestre Ciénaga de La Palmita e Isla de Pájaros, estado Zulia, Venezuela (Con permiso de: Vera et al., 2010).

La Reserva de Fauna Silvestre Ciénaga de La Palmita e Isla de Pájaros representa el área de transición entre la zona continental y el Lago de Maracaibo, donde recibe aportes del río Aurare (De Olivares, 1988), y del Lago de Maracaibo a través de tres entradas constituidas por caños sometidos a regímenes de marea. La precipitación media anual es de 400 a $500 \mathrm{~mm}$, con un patrón bimodal. El período seco se presenta de diciembre a marzo y de junio a agosto, y el lluvioso de septiembre a noviembre y de abril a mayo (Aguilera y Riveros, 1993).

Las máximas precipitaciones se registran en mayo y octubre, la evaporación oscila entre 2500 y $3000 \mathrm{~mm}$ anuales y la temperatura media anual entre 27,8 y $28,3^{\circ} \mathrm{C}$ (Aguilera y Riveros, 1993).

El área total de la RFS (2.525,85 ha), la cual se encuentra distribuida de la siguiente manera: dos mil quinientas hectáreas $(2500$ ha) de La Palmita y veinticinco hectáreas con ochenta y cinco áreas $(25,85 \mathrm{ha})$ de Isla de Pájaros (Gaceta Oficial de la República Bolivariana de Venezuela 2000). A su vez, en la superficie de 2.500 ha de la RFS se diferencian tres (3) zonas: el bosque de manglar (522 ha), la Ciénaga (855 ha) y el bosque xerófilo (1352,5 ha) (Gaceta Oficial de la República Bolivariana de Venezuela 2000). 
Obtención y procesamiento de las imágenes satelitales LANDSAT 8

En la investigación se emplearon imágenes satelitales LANDSAT 8 de los años 2013, 2014, 2015 y 2016 y se seleccionaron de acuerdo al cumplimiento de los siguientes criterios: abarcaron completamente el área de estudio y mostraron buena resolución temporal, es decir, no presentaron interferencias que impidieran la visualización del espacio físico.

Las imágenes satelitales LANDSAT 8 se descargaron del portal del servicio geológico de los Estados Unidos (USGS, 2013), se hizo una revisión de las diferentes imágenes y se solicitaron aquellas de interés.

A partir de cada imagen se desplegaron dieciséis archivos correspondientes a las bandas que componen una imagen LANDSAT 8 y se trabajó con las primeras 8 bandas.

Posteriormente se generó un vector del área de la Reserva de Fauna Silvestre y con la ayuda de éste, la zona de estudio se demarcó en cada imagen LANDSAT. El vector se superpuso sobre cada imagen LANDSAT y se eliminó la parte de la imagen fuera del mismo, quedando en cada caso únicamente el área de la RFS.

Finalmente se empleó el programa de procesamiento de imágenes ENVI para realizar cálculos de prueba del Índice Normalizado de Diferencias en la Vegetación (NDVI).

\section{Determinación de los índices de vegetación}

Los índices de vegetación se determinaron a partir del cálculo de los valores de NDVI (Índice Normalizado de Diferencias en la Vegetación o Índice de Vegetación de Antocianinas) mediante del programa ENVI Classic versión 5.0 (Environment for Visualizing Images).

\section{Muestreos de campo}

Los muestreos de campo se llevaron a cabo a través de exploraciones, recorridos vía acuática y terrestre, $\mathrm{y}$ actividades como: observaciones directas, toma de información por escrito, recolecta de muestras botánicas y toma de fotografías.

\section{Determinación taxonómica de las especies}

Las especies se determinaron taxonómicamente a partir de la experiencia profesional de los autores, la confrontación con material preservado del Herbario de la Universidad del Zulia "Omar Zambrano C." (HERZU) y de la bibliografía de Hoyos (1985) y Steyermark (1994). La nomenclatura de los epítetos se siguió de acuerdo a Hokche et al. (2008).

\section{Determinación de las unidades de paisaje}

La determinación de las unidades de paisaje se realizó a partir de los valores de NDVI obtenidos, los cuales se contrastaron con la información y los datos recabados durante los muestreos de campo (visitas y exploraciones in situ); todo esto contribuyó a disponer de una información más completa y concluyente. A través de este proceso se logró discriminar, diferenciar, separar e identificar las diferentes unidades de paisaje.

\section{Elaboración de mapas de las unidades de paisaje}

Finalmente, se realizaron clasificaciones que permitieron la elaboración de mapas de las unidades de paisaje de la Reserva de 
Fauna Silvestre Ciénaga de La Palmita e Isla de Pájaros usando los valores de NDVI y con ayuda del programa ENVI Classic versión 5.0. Además se emplearon colores distintos para la facilitar la diferenciación visual de cada una de las unidades de paisaje determinadas.

\section{RESULTADOS Y DISCUSIÓN}

La determinación de los índices normalizados de diferencias en la vegetación
(NDVI) permitió la identificación de siete (07) unidades de paisaje en la Reserva de Fauna Silvestre Ciénaga de La Palmita e Isla de Pájaros, Tabla 1 y Fig. 2.

Tabla 1. Índices de vegetación (NDVI) de las unidades de paisaje de la Reserva de Fauna Silvestre Ciénaga de La Palmita e Isla de Pájaros, estado Zulia, Venezuela.

\begin{tabular}{lcc}
\hline Unidades de paisaje (Up) & $\begin{array}{c}\text { Índice de vegetación } \\
\text { (NDVI) }\end{array}$ & $\begin{array}{c}\text { Identificación de las Up } \\
\text { (color) }\end{array}$ \\
\hline Espejo de agua de la ciénaga & $0,00-0,03$ & Celeste \\
$\begin{array}{l}\text { Borde ciénaga-porción terrestre } \\
\text { Suelos fangosos-desnudos }\end{array}$ & $0,04-0,08$ & Blanco \\
Comunidades xerófilas intervenidas & $0,09-0,16$ & Rojo \\
(arbustales y matorrales) & $0,17-0,24$ & Azul \\
$\begin{array}{l}\text { Comunidades xerófilas, espinosas } \\
\text { y semideciduas bajas }\end{array}$ & $0,25-0,28$ & Fucsia \\
$\begin{array}{l}\text { Comunidades de Avicennia germinans, } \\
\text { Conocarpus erectus y Laguncularia racemosa }\end{array}$ & $0,29-0,39$ & Amarillo \\
Comunidades de Rhizophora mangle & $0,4-0,55$ & Verde \\
\hline
\end{tabular}

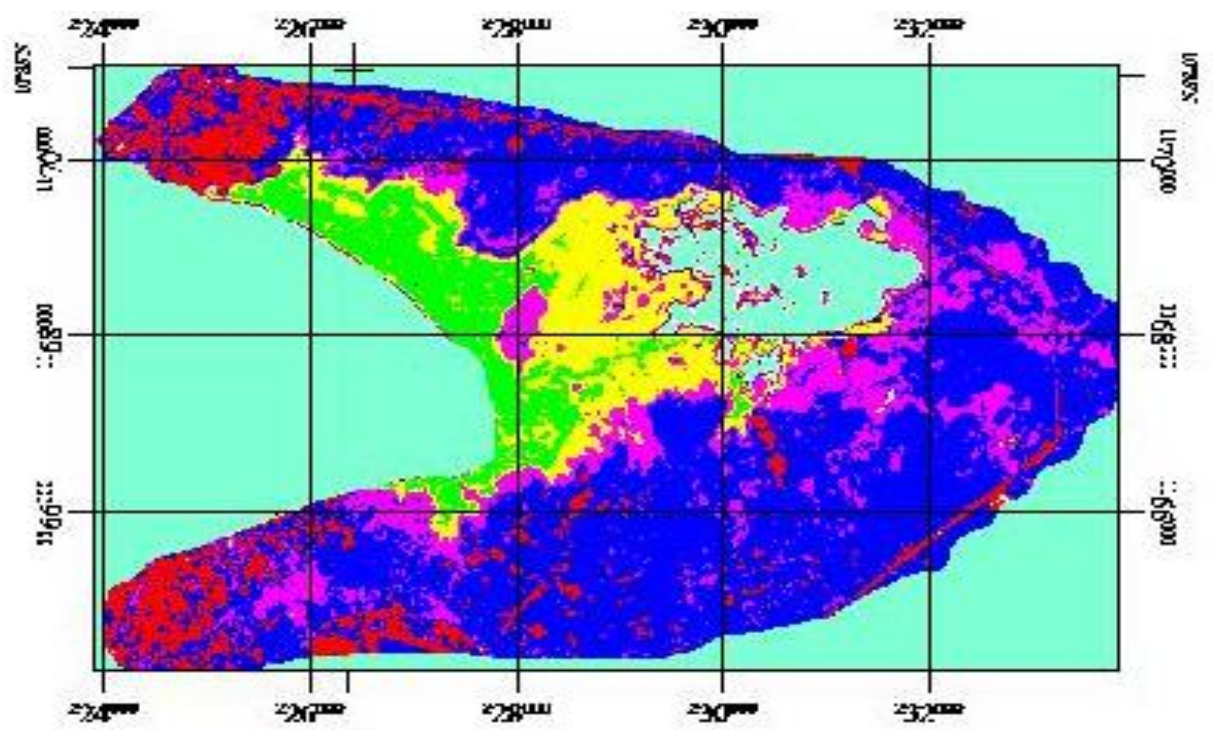

Figura 2. Unidades de paisaje de la Reserva de Fauna Silvestre Ciénaga de La Palmita e Isla de Pájaros. Imagen 058 (2014). LANDSAT 8. 


\section{Espejo de agua de la ciénaga}

La Ciénaga de La Palmita está formada por un cuerpo de agua somero (menor a $1 \mathrm{~m}$ de profundidad), cuyo aporte hídrico es suministrado por el río Aurare, Fig. 1. El reconocimiento de campo evidenció que la ciénaga presenta aguas turbias, lo cual se asocia a la descarga de sedimentos, al arrastre por escorrentía y a la escasa profundidad de la misma.

El valor muy bajo de NDVI $(0,00-0,03)$ para el espejo de agua de la Ciénaga de La Palmita, Tabla 1 y Fig. 2 (color celeste), indica que la superficie presenta muy poco vigor vegetal, es decir, que la imagen observada corresponde a zonas sin vegetación; tal como se ha reportado para los suelos desnudos, los cuerpos de agua y el material inerte en suspensión (Gómez Almonte, 2005; Meera Gandhi et al., 2015).

\section{Borde ciénaga-porción terrestre}

La unidad de paisaje borde ciénagaporción terrestre presentó un NDVI de 0,040,08 Tabla 1 y Figura 2 (color blanco) y la misma corresponde a la zona donde hace contacto el espejo de agua con el sustrato terrestre adyacente. Dichos valores se podrían relacionar con los NDVI de 0 a 0,1 correspondientes a los primeros indicios de productividad de acuerdo a lo reportado por Meera Gandhi et al. (2015).

Los valores de NDVI obtenidos para la unidad de paisaje borde ciénaga-porción terrestre también son comparables a los registrados para los humedales de la zona del río Paraná en Brasil con valores de NDVI que inician desde 0,07 (Borro et al., 2014) y para el fitoplancton de la estación Santa Rita, Costa Oriental del Lago de Maracaibo, estado Zulia cuyo NDVI fue de 0,086 (Morillo et al., 2018).

\section{Suelos fangosos-desnudos}

Los suelos fangosos-desnudos reciben esta denominación por tratarse de una unidad de paisaje que presenta un sustrato de lodo (fango) y materia orgánica acumulada del manglar en contacto con el agua de la ciénaga. Esta unidad de paisaje exhibió un NDVI de 0,09-0,16 Tabla 1 y Fig. 2 (color rojo), el cual a pesar de ser aun relativamente bajo, el mismo se asocia a la acumulación de capas de fitoplancton en su superficie.

A pesar de los valores muy bajos de NDVI señalados para las dos unidades de paisaje previas, para la Ciénaga de La Palmita se han reportado algunos géneros de fitoplancton como Chlorella, Closterium, Navicula, Nostoc, Oscillatoria, Pinnularia, Scenedesmus, Spirulina y Spirogyra (De Olivares, 1988). Es posible, que estos microorganismos fotosintéticos depositados en los suelos fangosos-desnudos, por acción del agua de la ciénaga, podrían incrementar su densidad celular (crecimiento) a partir de los nutrientes aportados por la materia orgánica alóctona del manglar, y de esta forma arrojar un valor de NDVI superior a los dos anteriores.

\section{Comunidades xerófilas intervenidas (arbustales y matorrales)}

Las comunidades xerófilas intervenidas forman parte del área de bosque xerófilo de 1.352,5 ha de la Reserva de Fauna Silvestre reseñado en la Gaceta Oficial de la República Bolivariana de Venezuela № 36.911 (2000), el cual rodea tanto al norte como al sur a la ciénaga y se ubica en la parte posterior del manglar.

Las comunidades xerófilas de la zona norte de la ciénaga muestran una gran modificación en su estructura. Dichas formaciones han estado expuestas a cambios en el uso del suelo y la cobertura vegetal, modificando el paisaje de tierras con vegetación leñosa xerófila a suelos para uso agrícola y de pastoreo. 
El bosque xerófilo está intervenido por diversas actividades antropogénicas como agricultura, ganadería, tala, caza y urbanización a las cuales se les atribuye los cambios en la cobertura vegetal, el uso del suelo, la composición florística, los rasgos fisonómicos y estructurales de la vegetación entre otras consecuencias.

Todas estas acciones han modificado el boque xerófilo primario convirtiéndolo en comunidades estructuralmente más simples y de menor riqueza florística, a las cuales se les puede denominar relictos de vegetación xerófila, arbustales o matorrales xerófilos intervenidos.

En tal sentido, Vera et al. (2009) han descrito para el poblado de Punta de Piedras, Municipio Miranda, estado Zulia, localidad muy cercana a la Reserva de Fauna Silvestre en estudio, un matorral xerófilo espinoso intervenido por quemas periódicas, deposición de desechos sólidos, caminerías para el tránsito de las personas y asentamientos poblacionales; originando la fragmentación de hábitats y la aparición de manchones (parches) de vegetación arbustiva-arbórea y de formaciones graminiformes 0 pastizales secundarios.

Estos argumentos sustentan y explican que dichas comunidades xerófilas intervenidas de la Ciénaga de La Palmita hayan arrojado un NDVI relativamente bajo, entre $0,17-0,24$, en relación a los valores de otras comunidades xerófilas determinadas en la presente investigación, Tabla 1 y Fig. 2 (color azul).

Además los valores de NDVI encontrados en esta investigación para las comunidades xerófilas intervenidas, son comparables a las registrados para la vegetación de tierras secas (clima árido, baja precipitación anual y altas temperaturas) circundante a los humedales del Complejo Acuífero Dalhousie (Dalhousie
Spring Complex) al sur de Australia, cuyo NDVI osciló entre 0,11-0,32 (White et al., 2016).

\section{Comunidades xerófilas, espinosas y semideciduas bajas}

La unidad de paisaje referente a las comunidades xerófilas, espinosas y semideciduas bajas, correspondió a la zona fitogeográfica denominada Isleta El Hicacal de 26 ha aproximadamente, y la misma se encuentra físicamente separada (delimitación espacial) del resto del bosque xerófilo de la Reserva de Fauna Silvestre (RFS), por el bosque de manglar que forma parte de esta misma ABRAE, Fig. 1. Es probable que la denominación de "isleta" que ha recibido esta zona haya sido precisamente por este estado de aislamiento o separación física de las otras formaciones vegetales adyacentes.

De acuerdo a estas características, la Isleta El Hicacal se considera un pequeño enclave xerofito que resultó separado del resto de la vegetación xerófila de la reserva posiblemente por el desarrollo y la expansión del bosque de manglar que finalmente lo rodeó, delimitó y aisló (Aranguren, comunicación personal).

Los trabajos de campo demostraron que el enclave xerofito de la Isleta El Hicacal, en general, está integrado por comunidades xerófilas, espinosas y semideciduas bajas. Sin embargo, los recorridos en la zona permitieron detectar áreas de herbazales halófitos (dada su vecindad con el bosque denso de manglar dominado por R. mangle y las áreas del ecotono), de pastizales, de cardonales-espinares y de comunidades deciduas.

Todos estos rasgos fisonómicos heterogéneos de la vegetación de la isleta El Hicacal permitieron definir a dicha zona fitogeográfica como un arbustal con una 
relativa "buena" conformación estructural y bajo nivel de intervención (impacto); situación está que se relaciona con su separación o asilamiento físico-geográfico de la acción humana.

Todo esto ha evitado acciones de perturbación o disturbios antropogénicos como tala, quema, urbanización, actividades agrícolas y pecuarias, entre otras; las cuales si han tenido lugar en la unidad de paisaje de comunidades xerófilas intervenidas (matorrales y arbustales).

El valor de NDVI de las comunidades xerófilas, espinosas y semideciduas bajas de la isleta El Hicacal osciló de 0,25-0,28 Tabla 1 y Fig. 2 (color fucsia), el cual coincide con lo señalado por Meera Gandhi et al. (2015) quienes han indicado que los arbustales y pastizales presentan valores moderados de 0,2 a 0,3. Igualmente, Petronelli et al. (2005) y Lovelock et al. (2017) han reportado que tanto la vegetación dispersa como los arbustales abiertos tienden a presentar valores de NDVI de 0,2 a 0,3; destacando además que las variaciones estacionales también pueden influir en que dichas formaciones vegetales muestren estos valores de NDVI.

Es posible que el bajo porte físicoestructural y el hábito de vida de las especies que integran algunas de las distintas comunidades vegetales presentes en la Isleta El Hicacal como los herbazales halófitos y los pastizales, sean las responsables de que esta unidad de paisaje presente dichos valores de NDVI, Tabla 1.

Aunado a ello, cabe destacar que el carácter deciduo o caducifolio de algunas especies como Astronium graveolens, Bulmesia arborea, Bursera simaruba, Bursera tomentosa, Cochlospermum vitifolium, Gyrocarpus americanus, Malphigia glabra, Pereskia guamacho, Pithecellobium dulce,
Pithecellobium forfex, Pseudobombax septenatum, Randia gaumeri, Ruprechtia ramiflora, Senna atomaria, Tabebuia serratifolia, entre otras presentes en la Isleta El Hicacal, caracterizadas por la pérdida de la biomasa foliar en durante el periodo de sequía, sea la explicación para que la vegetación de esta unidad de paisaje muestre los valores de NDVI registrados, Tabla 1 y Fig. 2.

\section{Comunidades de Avicennia germinans, Conocarpus erectus y Laguncularia racemosa}

Las comunidades de Avicennia germinans, Conocarpus erectus y Laguncularia racemosa mostraron un NDVI entre 0,29-0,39 Tabla 1y Fig. 2 (color amarillo). Estos valores son más bajos y distinguibles de los obtenidos para las comunidades de R. mangle, Tabla 1, lo cual se puede explicar para el caso de A. germinans en que este mangle presenta un color verde pálido (grisáceo) en sus hojas. Esto se asocia con la reflectancia del dosel y en el hecho que el Índice Normalizado de Diferencias en la Vegetación (NDVI) básicamente es directamente proporcional al verdor de la vegetación y a la densidad de clorofila (Meera Gandhi y col., 2015).

Este mismo argumento aplica para C. erectus y L. racemosa, especies que muestran un color verde pálido amarillento en sus órganos foliares. Además, estos dos últimos mangles están representados por arbustos y árboles de bajo porte (altura media de $8 \mathrm{~m}$ aproximadamente), con follaje menos denso, menor cantidad de organismos y además se encontraron individuos muertos de ambas especies en algunas áreas muestreadas cerca de la ciénaga. 
Las características similares señaladas para los mangles A. germinans, C. erectus y $\mathrm{L}$. racemosa, y para las comunidades que estos conformaron, podrían ser las responsables del hecho que dichas formaciones vegetales no mostraron valores de NDVI (diferentes) y en lugar de ello el programa ENVI, durante el análisis de las imágenes satelitales, las agrupó en una sola unidad de paisaje sin realizar una discriminación para separarlas. Sin embargo, los trabajos y las exploraciones de campo (in situ) confirmaron que realmente los valores de NDVI entre 0,29-0,39 correspondieron a comunidades de A. germinans, C. erectus y L. racemosa.

Además de los expuesto, Waas y Nababan (2010) reportaron que la vegetación "relativamente densa" de manglar se caracteriza por mostrar valores de NDVI entre $0,3-0,4$, los cuales coinciden con los señalados para las comunidades de A. germinans, C. erectus y L. racemosa de esta investigación, Tabla 1.

\section{Comunidades de Rhizophora mangle}

La exploraciones de campo demostraron que las comunidades de R. mangle están integradas casi de forma monoespecífica por arboles de mangle rojo, de altura promedio de $15 \mathrm{~m}$ aproximadamente, con un dosel cerrado a semicerrado (bóveda casi continua) y pocas aberturas o claros (gaps). A partir de estas observaciones, producto de los muestreos, y tomando en consideración las características estructurales mencionadas, se podría sugerir que se trata de un bosque de manglar maduro.

Esta comunidad de manglar, de acuerdo a la Gaceta Oficial de la República Bolivariana de Venezuela № 36.911 (2000), representa uno de los cuatro espacios bióticos que integran la Reserva de Fauna Silvestre
Ciénaga de La Palmita e Isla de Pájaros y está constituida por un denso bosque de 522 ha.

Las comunidades de R. mangle (mangle rojo) presentaron el NDVI más alto $(0,4-0,55)$ Tabla 1 y Fig. 2 (color verde), en comparación con el resto de las formaciones vegetales descritas en esta investigación Esto se puede explicar a partir de los rasgos estructurales señalados anteriormente dado que según Waas y Nababan (2010) la vegetación muy densa de manglar se caracteriza por presentar valores de NDVI mayores a 0,4.

En apoyo a la explicación señalada, R. mangle posee un verdor foliar intenso $\mathrm{y}$ brillante, que además de los argumentos planteados, éste puede tener influencia en los valores de NDVI presentados. La interpretación realizada tiene sustento en los reportes señalados por Jarchow et al. (2016) quienes sostienen que el NDVI responde a los órganos fotosintéticamente activos, aumentando a medida que incrementa la cantidad de biomasa verde.

Por otra parte, los manglares son bosques costeros tropicales siempre verdes y en el presente estudio las comunidades de $\mathrm{R}$. mangle mostraron un NDVI que alcanzó 0,55; el cual es un valor muy cercano al descrito por Meera Gandhi et al. (2015) quienes señalaron que valores elevados de NDVI entre 0,6-0,8 son indicadores de ecosistemas como los bosques lluviosos tropicales.

Santos et al. (2012) reportaron que el hábitat tipo 3 de vegetación del manglar del Estuario del río São Francisco al noreste de Brasil mostró un NDVI entre 0,45-0,57. Dicha comunidad vegetal también se caracterizó por un cierre del dosel de 50 a 75\% (cobertura densa), con pequeños claros o aperturas (gaps), una alta presencia de árboles de R. mangle y además constituyó el hábitat dominante en el área de estudio. Los rasgos 
fisonómico-estructurales mencionados son muy parecidos a los señalados para las comunidades de R. mangle de la RFS Ciénaga de La Palmita e Isla de Pájaros; lo que reafirma la explicación de haber resultado la unidad de paisaje con el mayor valor de NDVI.

\section{CONCLUSIONES}

La Reserva de Fauna Silvestre Ciénaga de La Palmita e Isla de Pájaros presenta unidades de paisaje heterogéneas como comunidades xerófilas intervenidas (relictos de vegetación xerófila) afectadas por perturbaciones y con valores de NDVI relativamente bajos; y comunidades de $R$. mangle con NDVI comparativamente altos, lo que sugiere que estas últimas se encuentran en buen estado de conservación.

\section{Agradecimientos}

Al Centro de Investigación del Agua de la Facultad de Ingeniería por el soporte técnico brindado y al Doctorado en Ciencias Agrarias de la Facultad de Agronomía de la Universidad del Zulia por el apoyo logístico prestado para los trabajos de campo.

\section{REFERENCIAS}

Aguilera, M. y Riveros, J. (1993). Diagnóstico preliminar de la ciénaga de La Palmita, municipios Miranda y Santa Rita e Isla de Pájaros, municipio Santa Rita. PROFAUNA. MARNR. Maracaibo

Borro, M., Morandeira, N., Salvia, M., Minotti, P., Perna, P. y Kandus, P. (2014). Mapping shallows lakes in a large South American floodplain: a frequency approach on multitemporal Landsat TM/ETM data. Journal of Hydrology, 512, 39-52

Coty, J. (1995). Vertebrados terrestres de la Ciénaga de La Palmita, Costa Oriental del Lago de Maracaibo, Venezuela. Trabajo Especial de Grado. Licenciatura en Biología. Facultad Experimental de
Ciencias. Universidad del Zulia, Maracaibo, Venezuela

De Olivares, D. M. (1988). Caracterización ecológica del manglar de la ciénaga Las Palmitas. Bol. Centro Invest. Biol., 17, 91119

Ewel, J. y Madriz, A. (1968). Zonas de vida de Venezuela. Ministerio de Agricultura y Cría, Ediciones del Fondo Nacional de Investigaciones Agropecuarias. Editorial Sucre, Caracas, Venezuela

Gaceta Oficial de la República Bolivariana de Venezuela № 36.911. (2000). Decreto № 730 sobre la creación de la Reserva de Fauna Silvestre Ciénaga de La Palmita e Isla de Pájaros. Caracas. Venezuela

Gómez Almonte, M. K. (2005). Índices de vegetación en áreas del bosque seco del noroeste del Perú a partir de imágenes satelitales. Tesis para optar el título de Ingeniero Industrial $\mathrm{y}$ de Sistemas, Facultad de Ingeniería, Universidad de Piura

González-Bencomo, E. y Borjas, J. (2003). Inventario de la ictiofauna de la ensenada de La Palmita, estrecho del Lago de Maracaibo, Venezuela. Bol. Centro Invest. Biol., 37(2), 83-102

Jarchow, C. J., Nagler, P. L. y Glenn, E. P. (2016). Greenup and evapotranspiration following the Minute 319 pulse flow to Mexico: An analysis using LANDSAT 8 Normalized DifferenceVegetation Index (NDVI) data. Ecological Engineering. Consultado 20 Noviembre de 2018. Disponible en: http://dx.doi.org/10.1016/j.ecoleng.2016. $\underline{08.007}$

Hokche, O., Berry, P. E. y Huber, O. (2008). Nuevo catálogo de la flora vascular de Venezuela. Fundación Instituto Botánico de Venezuela Dr. Tobías Lasser, Caracas, Venezuela

Hoyos, J. (1985). Flora de Isla de Margarita, Venezuela. Monografía, № 34, Fundación de Ciencias Naturales La Salle. Caracas, Venezuela 
Lovelock, C. E, Feller, I. C., Reef, R., Hickery, S. y Ball, M. C. (2017). Mangrove dieback during fluctuating sea levels. Scientific Reports, 7(1680), 1-8. Doi: 10.1038/s41598-017-01927-6

Medina, E. y Barboza, F. (2006). Lagunas costeras del Lago de Maracaibo: distribución, estatus y perspectivas de conservación. Ecotropicos, 19(2), 128-139

Meera Gandhi, G., Parthiban, S., Nagaraj, T. y Christy, A. (2015). NDVI: Vegetation change detection using remote sensing and gis - A case study of Vellore District. Procedia Computer Science, 57, 1199-1210

Morillo Díaz, G., Aldana Villasmil, G. J., Pineda, A. y Royero Perozo, G. A. (2018). Estimación de clorofila a en el Lago de Maracaibo, Venezuela utilizando imágenes LANDSAT 8. Revista MAPPING, 27(188), 414

Pettrorelli, N., Vik, J. O., Mysterud, A., Gaillard J. M., Tucker, C. J. y Stenseth, N. C. (2005). Using the satellite-derived NDVI to assess ecological responses to environmental change. TRENDS in Ecology and Evolution, 20(9), 503-510, doi:10.1016/j.tree.2005.05.011

Pietrangeli, M. A., Villarreal, A. y Gil, B. (2011). Florística de las comunidades forestales de zonas aledañas al embalse Pueblo Viejo (Burro Negro), estado Zulia, Venezuela. Bol. Centro Invest. Biol., 45(3), 237-286

Powel, S., Jakeman, A. y Crokee, B. (2014). Can NDVI response indicate the effective flood extent in macrophyte dominate floodplain wetlands? Ecological indicators, 486-493

Santos, L. C. M., Bitencourt, M. D., Koedam, N. y Dahdouh-Guebas, F. (2012). Discrimination of mangrove vegetation habitat types (Northeast Brazil) using CBERS-2B images and the Normalized Difference Vegetation Index (NDVI). En F. Dahdouh-Guebas y B. Satyanarayana. (Eds.), Proceedings of the International Conference 'Meeting on Mangrove ecology, functioning and Management - MMM3' (57: pp. 164). Galle, Sri Lanka, 2-6 July 2012. VLIZ Special Publication.
Recuperado de http://www.marinespecies.org/imis.p hp? module $=$ ref \&refid $=223772$

Steyermark, J. (1994). Flora del Parque Nacional Morrocoy. Editado por: Bruno Manara. Fundación Instituto Botánico de Venezuela y Agencia Española de Cooperación Internacional, Retolit C.A., Caracas, Venezuela

U. S. Geologic Survey (USGS). (13 de Abril de 2013). Earth Resources Observation and Science (EROS) Center. Consultado el 25 de Noviembre de 2019, de U. S. Ge4ologic Survey (USGS). Disponible en: https://eros.usgs.gov/

Valbuena, C. A., Sánchez, A. y Portillo Quintero, C. (2011). Extensión y patrones fenológicos de bosques secos de la planicie de Maracaibo, utilizando sensores remotos. Informe Anual del Instituto Venezolano de Investigaciones Científicas (IVIC), Altos de Pipe, Venezuela. Consultado: 15 de Enero de 2020. Disponible en: www.ivic.gob.ve

Vera, A., Martínez, M., Ayala, Y., Montes, S. y González, A. (2009). Florística y fisonomía de un matorral xerófilo espinoso intervenido en Punta de Piedras, Municipio Miranda, Estado Zulia, Venezuela. Rev. Biol. Trop., 57(1-2), 271281

Vera, A., Martínez, M. y Nava, W. (2011a). Hemiparásitas de las especies de mangle de la Ciénaga de La Palmita, estado Zulia, Venezuela. Rev. Fac. Agron. (LUZ), 28(Supl.1), 110-119

Vera, A., Martínez, M., Parra, Y., Maldonado, R. y Carvajal, N. (2014). Plantas hemiparásitas y hospederas presentes en el bosque xerófilo de la Ciénaga de La Palmita, estado Zulia, Venezuela. Rev. Fac. Agron. (LUZ), (Supl.1), 213-223

Vera, A., Nozawa, S., Villarreal, A. y Martínez, M. (2011b). Estudio preliminar de las gramíneas de la Ciénaga de La Palmita, estado Zulia, Venezuela. Rev. Fac. Agron. (LUZ), 28(Supl.1), 120-128

Vera, A., Villarreal, A. y Martínez, M. (2010). Composición florística de cuatro ambientes en la ciénaga de La Palmita, 
estado Zulia, Venezuela. Acta Bot. Venez., 33(1), 23-34

Waas, H. J. D. y Nababan, B. (2010). Pemetaan dan analisis index vegetasi mangrove di Pulau Saparua, Maluku Tengah. E-Jurnal Ilmu dan Teknologi Kelautan Tropis, 2(1), 50-58
White, D. C., Lewis, M. M., Green, G. y Gotch, T. B. (2016). A generalizable NDVI-based wetland delineation indicator for remote monitoring of groundwater flows in the Australian Great Artesian Basin. Ecological Indicators, 60, 1309-1320 